\section{Vitamin-D-Mangel im Sonnenland}

\begin{abstract}
uviel Sonne verursacht Hautkrebs.
Vor allem australische Dermatologen sind in Sonnenvermeidungskampagnen zum Schutz der Gesundheit engagiert und gehen mit gutem Beispiel voran - vielleicht aber nicht ganz in die richtige Richtung. In einer einigermaßen repräsentativen Untersuchung fand man bei $83 \%$ der Hautärzte in der Region Melbourne einen deutlichen Vitamin-DMangel (Hydroxy-Vitamin-D-Spiegel unter $50 \mathrm{nmol} / \mathrm{l})$. Die Patienten der untersuchten Hautärzte wiesen höhere Spiegel auf, wenngleich auch bei ihnen häufig nur eine suboptimale Vitamin-D-Versorgung vorlag. Niedrige Vitamin-DSpiegel sind nicht nur schlecht für die
\end{abstract}

\section{Hautkrebs im Nagelstudio}

B ei der Nagelkosmetik werden die behandelten Fingernägel oft mit UVLicht bestrahlt, um sie zu härten. Dass dies ernstzunehmende Langzeitfolgen haben kann, zeigen zwei Fälle von Frauen aus Texas. Die erste Patientin wies ein spinozelluläres Karzinom in situ im Bereich der dorsomedialen Spitze des rechten Zeigefingers auf. Durch Sonnenlicht induzierte Schäden des Gesichts oder des sonstigen Körpers bestanden bei ihr nicht. Bei der zweiten Patientin traten im zeitlichen Verlauf mehrere spinozelluläre Karzinome der
Handrücken sowie des linken Zeigefingers (dorsaler Bereich) auf. Beide Frauen hatten über viele Jahre regelmäßig ihre Nägel behandeln und mit UV-A bestrahlen lassen. Sie waren beruflich keiner Sonneneinstrahlung ausgesetzt. Die Patientinnen hatten keine vorhergehenden Infektionen mit humanen Papillomviren, auch die Familienanamnese bezüglich Hautkrebs war unauffällig.

MacFarlane DF et al. Arch Dermatol 2009; 145: 447-9

\title{
Erythema ab igne durch einen Laptop
}

in 26-jähriger Mann präsentierte sich in einer Pariser Klinik mit retikulär angeordneten, fleckförmigen, bräunlichen Pigmentierungen an den Vorderseiten der Oberschenkel. Der junge Mann hatte sich einige Zeit zuvor einen Laptop gekauft. Mehrere Stunden pro Tag hatte er damit gearbeitet, wobei er das Gerät jeweils auf seinen Oberschenkeln abgelegt hatte. Die Ärzte stellten die Diagnose eines Erythema ab igne. Dieses Krankheitsbild wird vor allem im Schienbeinbereich durch zu nahes Sitzen am offenen Kaminfeuer oder an der Bauchdecke durch Wärmflaschen verursacht. In diesem Fall war ein Laptop die Ursache: Die Geräte können Betriebstemperaturen von bis zu $50{ }^{\circ} \mathrm{C}$ erreichen und sollten daher nicht länger auf der Körperoberfläche zu liegen kommen.

Bachmeyer C et al. J Eur Acad Dermatol Venereol 2009; 23: 736-7
Notfall nach Notfallpille

E ine 28-jährige Patientin stellte sich - in einer Klinik mit Fieber, Hautexanthem und Konjunktivitis vor. Die Suche nach einer bakteriellen oder viralen Genese verlief erfolglos. Die Anamnese blieb unauffällig - bis auf die Einnahme einer Notfallpille mit Levonorgestrel und Ethinylestradiol wenige Tage zuvor. Im weiteren Verlauf entwickelten sich Blasen, die etwa $80 \%$ der Körperoberfläche bedeckten. Die Dermatologen stellten die Diagnose einer toxischen epidermalen Nekrolyse. Eine Behandlung mit intravenösen Immunglobulinen musste wegen Leukopenie und Pleuraergüssen abgebrochen werden, trotzdem kam es zum Abklingen der Symptome. Die Patientin konnte nach zwei Wochen entlassen werden.

af

Weinberger $\mathrm{CH}$ et al. J Am Acad Dermatol 2009; 60: 708-9

\section{Asthma in der Fischfabrik}

- ine 54Arbeiterin klagte über Ekzeme an Händen und Unterarmen, weiter berichtete sie über Atemnot. Beide Er- ${ }^{{ }^{2}} \mathrm{Ch}_{\mathrm{n}_{\mathrm{g}}}$ krankungen traten bei der Arbeit im fischverarbeitenden Gewerbe auf, bei der sie Arbeitshandschuhe und Mundschutz nur gelegentlich trug. Sowohl Pricktest als auch Patchtest zeigten starke Reaktionen gegen Anisakis simplex, einen Fadenwurm, der Fische befällt. Das Gesamt-IgE war erhöht, jedoch ohne Erhöhung des spezifischen IgE gegen Anisakis simplex. Konstantes Tragen von Handschuhen bewirkte nur eine leichte Besserung der Hautbeschwerden. Alle Symptome verschwanden aber, als die Patientin ihre Tätigkeit in der Fischindustrie aufgab.

af

Barbuzza 0 et al. Contact Dermatitis 2009; 60: 239-40 